\title{
Comets: extremal states and their observational manifestations
}

\author{
Ibadov Subhon ${ }^{1}$ and Firuz S. Ibodov ${ }^{2}$ \\ ${ }^{1}$ Institute of Astrophysics, Dushanbe, Tajikistan \\ email: ibadovsu@yandex.ru \\ ${ }^{2}$ Lomonosov Moscow State University, Moscow, Russia \\ email: mshtf@sai.msu.ru
}

\begin{abstract}
Current status and prospects of further investigations are considered for: 1.Anomaly distribution of Na-atoms emission in the cometary heads; 2. Mechanisms of X-ray generation in comets; 3. Evolution of sungrazing comets near the Sun.
\end{abstract}

Keywords. Comets:general; Na-emission, anomaly distribution; X-rays, high-temperature plasma, solar wind, multicharge ions; cometary impacts, the Sun.

Modern models of cometary nuclei and their atmospheres as well as parameters of orbital motion of comets indicate the presence of extremal states in such objects of the Solar System. 1. The temperature distribution of the cometary gas has the minimum, in the range of 5-100 K, in the inner coma. This may cause the observed anomaly distribution of emission of Na-atoms in the heads of such bright comets like comet Mrkos 1957 d/1957 V and, possibly, in jets of comet Halley 1986 III, due to effect of depression of the temperature of cometary dust by extremely cold and dense gas in the near-nuclear region. 2. High-velocity collisions between cometary and interplanetary dust particles can lead to generation of short-living high-temperature $(>100000 \mathrm{~K})$ plasma clots in the dust coma of comets, that will be accompanied by generation of soft, 0.1-1 keV, X-rays from comets, as well as multicharge ions, in the inner heliosphere. 3. Impacts of sungrazing comet nuclei with the Sun with velocities more $600 \mathrm{~km} / \mathrm{s}$ will be accompanied, due to preliminary aerodynamic crushing of comet nuclei and transversal expansion of crushed matter, by impulsive generation of hot sub-photospheric plasma and "blast" shock wave induced ejection of hot plume into space above the solar chromosphere. Observations of mentioned phenomena by modern large telescopes, having high spatio-temporal resolutions, from ground-based and space observatories, together with in situ measurements during space missions in the future, are of interest not only in the study of comets but also in related fields.

\section{References}

Dobrovolsky, O. V. 1966, in Comets, Nauka, Moscow, 288

Greenstein, F. L. \& Arpigny, C. 1962, Astrophys. J. 135, 892

Grigorian, S. S., Ibadov, S., \& Ibodov, F. S. 2000, Dokl. Akad. Nauk 374, 40 [Engl. Transl.: Phys.-Dokl. 45, 463]

Ibadov, S. 1990, Icarus 86, 283

Ibadov, S. 1996, in Physical Processes in Comets and Related Objects, Cosmosinform Publ. Comp., Moscow, 181

Ibadov, S. 2000, Astron. Astrophys. Transac. 18:6, 799

Ibadov, S. 2011, in: Advances in Plasma Astrophysics, Cambridge Univ. Press, 76

Ibodov, F. S. \& Ibadov, S. 2011, in: Advances in Plasma Astrophysics, CUP, 119 